\title{
IX Draconis, ER UMa-type dwarf nova
}

\author{
M. Otulakowska-Hypka ${ }^{1}$, A. Olech ${ }^{1}$, E. de Miguel $^{2}$, A. Rutkowski ${ }^{3,4}$, \\ R. Koff ${ }^{5}$, K. Bakowska ${ }^{1,6}$ \\ ${ }^{1}$ N. Copernicus Astronomical Center, Polish Academy of Sciences, Warsaw, Poland \\ email: magdaot@camk.edu.pl \\ ${ }^{2}$ Dep. de Fisica Aplicada, Facultad de Ciencias Experimentales, Universidad de Huelva, Spain \\ ${ }^{3}$ TUBITAK National Observatory, Akdeniz University Campus, Antalya, Turkey \\ ${ }^{4}$ Astronomical Observatory, Jagiellonian University, Kraków, Poland \\ ${ }^{5}$ Center for Backyard Astrophysics, Antelope Hills Observatory, Bennett, CO, USA \\ ${ }^{6}$ Astronomical Observatory, Faculty of Physics, A. Mickiewicz University, Poznań, Poland
}

\begin{abstract}
We present results of our world-wide observing campaign which was dedicated to one of the most active dwarf novae, IX Draconis. We investigated photometric behaviour of the system to derive its basic parameters. This study is important in the context of our understanding of the accretion process of IX Draconis, as well as other active cataclysmic variables, especially those from the still poorly studied type of ER UMa stars.
\end{abstract}

Keywords. binaries: close - stars: cataclysmic variables, dwarf novae, individual: IX Draconis

\section{Introduction}

One of the types of cataclysmic variables are SU UMa stars, which are dwarf novae with very short orbital periods, $P_{\text {orb }}<2.5 \mathrm{~h}$. In such systems a white dwarf is the primary star, and a low mass main sequence star, filling its Roche lobe, is the secondary. The main observational feature of SU UMa stars is, beside normal outbursts, presence of superoutbursts with additional "tooth-shape" light curve modulations, called superhumps. The most active among SU UMa-type stars are ER UMa-type stars (Kato et al. 1995). These are objects with extremely short supercycle and normal cycle lengths. They are probably standard SU UMa stars with only one difference - larger activity and luminosity caused by their higher mass transfer rates, as was shown by Osaki (1995).

Up to now we know only five stars belonging to the ER UMa-type of stars: ER UMa (Kato et al. 1995), V1159 Ori (Patterson et al. 1995, Szkody et al. 1999), RZ LMi (Olech et al. 2008), DI UMa (Rutkowski et al. 2009), and IX Dra (Olech et al. 2004). Therefore, studying each of them is extremely important.

\section{Overview}

We report results of our world-wide observing campaign dedicated to IX Dra. Six observers used eleven small telescopes (with diameters of main mirrors up to 1 meter) in four different countries to almost continuously monitor the object over two months in 2010. The result of these observations is a beautiful, well covered light curve which shows two superoutbursts and several normal outbursts in between them.

In order to measure basic outburst properties of the system, we carried out analyses of the light curve, power spectra and $O-C$ diagrams.

One of the most important findings is the fact that the supercycle length of IX Dra continually increases during the past twenty years and is now equal to $58.5 \pm 0.5 \mathrm{~d}$. See Fig. 1. 


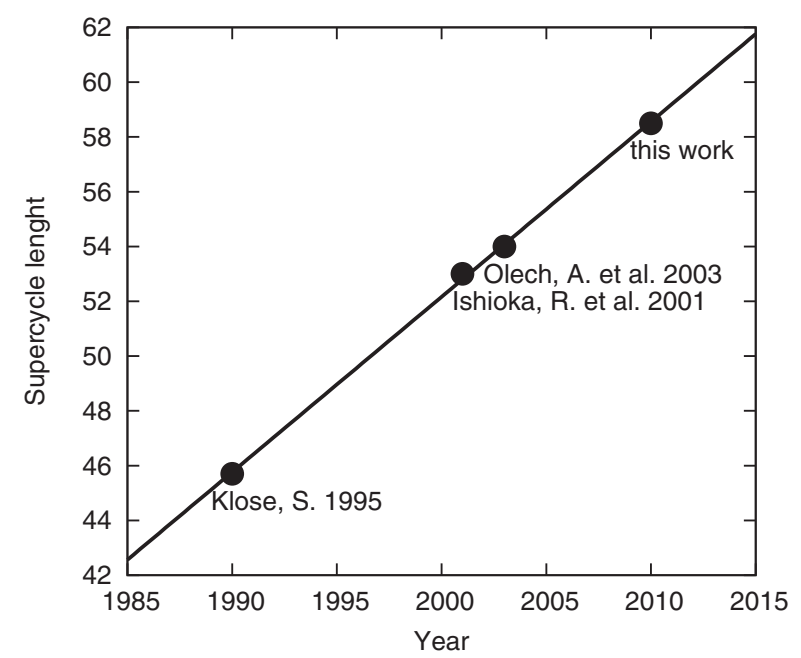

Figure 1. The upward trend of the supercycle length of IX Dra during the past twenty years.

Data points are taken from Klose 1995, Ishioka et al. 2001, and Olech et al. 2004.

We detected double humps during superoutburst, with the secondary maximum peaking near the end of the superoutburst. They appeared with the same superhump period, which is constant for IX Dra over the last twenty years and is equal to $P_{s h}=$ $0.066982(36) \mathrm{d}$.

Based on our observations, there are two possible values of the orbital period. The more likely is $0.06641(3) \mathrm{d}$, which is in agreement with Olech et al. 2004 and our $O-C$ analysis $(0.06646(2))$.

Normal outbursts are irregular. The interval between two successive normal outbursts is $3.1-4.1$ days.

\section{Acknowledgements}

The project was supported by Polish National Science Center grant awarded by decision number DEC-2012/04/S/ST9/00021 to AR, and Polish National Science Center grant awarded by decision number DEC-2011/03/N/ST9/03289 to MOH.

\section{References}

Ishioka, R., Kato, T., Uemura, M. et al. 2001, PASJ, 53, L51

Kato, T. \& Kunjaya, C. 1995, PASJ, 47, 163

Klose, S. 1995, ApJ, 446, 357

Olech, A., Zloczewski, K., Mularczyk, K., Kedzierski, P. et al. 2004, AcA, 54, 57

Olech, A., Wisniewski, M., Zloczewski, K., Cook, L. M. et al. 2008, AcA, 58, 131

Osaki, Y. 1995, PASJ, 47, L11

Patterson, J., Jablonski, F., Koen, C., O’Donoghue, D. et al. 1995, PASP, 107, 1183

Rutkowski, A., Olech, A., Wiśniewski, M., Pietrukowicz, P. et al. 2009, A\&AA, 497, 437

Szkody, P., Linnell, A., Honeycutt, K., Robertson, J. et al. 1999, ApJ, 521, 362 